\title{
Incorporation of Viscoelastic Loss into the Plane Wave Expansion Approach to Modelling Composite Transducers
}

\author{
Leigh-Ann Orr and Anthony J. Mulholland \\ Department of Mathematics, \\ University of Strathclyde, \\ Glasgow, UK G1 1XH
}

\author{
Richard L. O'Leary and Gordon Hayward \\ The Centre for Ultrasonic Engineering, \\ Department of Electrical and Electronic Engineering, \\ University of Strathclyde, \\ Glasgow, UK G1 1XW
}

\begin{abstract}
The Plane Wave Expansion (PWE) method has been proposed as a theoretical model for periodic composite ultrasonic transducers. This paper extends previous work by importantly including viscoelastic loss in the material parameters. Some of the issues with model formulation, such as ill-conditioning in the large matrices, have been addressed through parameter scaling and Tikhonov regularisation. Identification of each mode of vibration has been carried out by visualising the spatial and temporal profiles of the displacement, electrical potential and Poynting vector. A comparison between the theoretical predictions and experimental data from a piezoelectric composite device is presented. The effect that the elastic properties of the passive phase have on device performance is also investigated. It is found that high shear attenuation in the passive phase gives rise to a large frequency stop band gap around the fundamental thickness mode.
\end{abstract}

\section{INTRODUCTION}

Piezoelectric composite transducers are generally accepted as the design of choice in many biomedical, sonar and nondestructive testing applications ([1], [2]). The most frequently used designs are made by dicing the ceramic into a series of pillars and then filling the void with a passive polymer phase [3]. A problem with this architecture is the presence of unwanted waves, which are generated between the adjacent pillars (inter-pillar modes) or within the pillars (intra-pillar modes), interfering with the piston like behaviour of the main thickness mode [4]. It has been suggested that a passive material with a low transverse coupling could damp out these unwanted modes ([4], [5]). Theoretical modelling of the transducer can enhance the understanding of the underlying physics and help in the prototyping of new designs ([6], [7], [8], [9]). A complementary approach to Finite Element (FE) modelling is the plane wave expansion (PWE) method introduced by Wilm et al [10]. This paper extends this model by incorporating loss into the formulation; vital for accurate representation of transducer behaviour since it has been shown to affect crosstalk between adjacent ceramic pillars. The next section details the geometry of the transducer, its description in terms of a Fourier analysis and a brief outline of the PWE method. This section follows the derivation of Wilm et al [10] and so only a brief outline is given. The inclusion of viscoelastic loss into the model is described, followed by a discussion on the use of scaling and regularisation in the method's implementation. A comparison between the PWE method, FE modelling and experimentally measured transducer behaviour is reported. Dispersion curves, electrical impedance plots and a modal analysis using displacement and Poynting vector profiles are used to discuss the operating characteristics. Finally, a composite transducer with a high shear loss passive phase is analysed and the results show an improvement in the device's stop band gap surrounding the fundamental thickness mode.

\section{Formulation of the Model}

The model is configured for periodic 2-2 and 1-3 composites, with thickness in the $x_{3}$ direction. Due to the periodicity of the structure, the material constants, such as the density $\rho$, the elasticity tensor $c_{i j k l}$, the piezoelectric stress tensor $e_{i j k}$ and the permittivity tensor $\epsilon_{i j}$, are expanded as spatial Fourier series in the $x_{1}-x_{2}$ plane. For the 1-3 composite structure, the material constants only depend on $x_{1}$ and $x_{2}$ and hence the dependent variables propagating within these periodic structures can be expressed as Floquet series. The physical model of the transducer is provided by the piezoelectric constitutive equations together with Newton's second law and Gauss's law for dielectric media [11]

$$
\begin{aligned}
T_{i j} & =c_{i j k l} u_{k, l}+e_{l i j} \phi_{, l} \\
D_{i} & =e_{i k l} u_{k, l}-\epsilon_{i l} \phi_{, l} \\
\rho \frac{\partial^{2} u_{j}}{\partial t^{2}} & =T_{i j, i} \\
D_{i, i} & =0 .
\end{aligned}
$$

Equations (1) to (4) constitute 16 equations in the 16 unknowns which are the stresses $T_{i j}$, the displacements $u_{k}$, the electric potential $\phi$ and the electrical displacements $D_{i}$. By substituting the Floquet representation into the model, a generalised eigenvalue problem arises in the thickness direction 
wavenumber $k_{3}$. Lengthy algebraic manipulations lead to the following approximation for the dependent variables ([10], [12])

$$
\begin{aligned}
\left(\begin{array}{c}
u(r, t) \\
t_{3}(r, t)
\end{array}\right) & =e^{\jmath\left(\omega t-k_{1} x_{1}-k_{2} x_{2}\right)} \times \\
\sum_{q=1}^{(2 N+1)^{2}} e^{-\jmath G^{q} \cdot r} & \left(\sum_{r=1}^{8(2 N+1)^{2}} A^{(r)} e^{-\jmath k_{3}^{(r)} x_{3}}\left[\begin{array}{l}
u^{q} \\
t_{3}^{q}
\end{array}\right]^{(r)}\right),
\end{aligned}
$$

where $r=\left(x_{1}, x_{2}, x_{3}\right), t$ is time, $\omega$ is the angular frequency, $k=\left(k_{1}, k_{2}, k_{3}\right)$ is the wave vector, the generalized displacement field is $u=\left(u_{1}, u_{2}, u_{3}, \phi\right)$, the generalized stress vector is $t_{i}=\left(T_{i 1}, T_{i 2}, T_{i 3}, D_{i}\right)$, the $8(2 N+1)^{2}$ eigenvalues $k_{3}^{(r)}$ have corresponding eigenvectors $\left[\begin{array}{ll}u^{q} & t_{3}^{q}\end{array}\right]^{(r)}$ and relative amplitudes $A^{(r)}$, and $N$ is the number of Fourier coefficients. Energy distribution within the transducer can be used to clarify particular types of modes in conjunction with examining the profiles of the displacements, stresses and electric potential. To examine the energy distribution in the device the Poynting vector $\left(P_{j}\right)$ is used. This can be shown to be [12]

$$
P_{j}=-\jmath \omega\left(c_{i j k l} u_{k, l}+e_{l i j} \phi_{, l}\right) u_{i}+\jmath \omega \phi\left(e_{j k l} u_{k, l}-\epsilon_{j l} \phi_{, l}\right) .
$$

The method is sufficiently general to cope with a wide range of boundary conditions. For simplicity the mechanical boundary condition of a stress free plate and the electrical boundary condition of a fixed electrical potential at the top and bottom of the transducer are employed when deriving dispersion relationships. An alternative electrical boundary condition of continuity and smoothness in the electrical potential at the electroded surfaces is employed for the calculation of the relative amplitudes and hence the dependent variables, using equation (5). One advantage of studying piezoelectric composites is that the electrical operating characteristics provide an alternative means of deriving the dispersion curves, whereby the resonant modes are signified by maxima in the real part of the admittance $(Y)$. Using continuity of the electrical potential at the front interface gives [10]

$$
\begin{gathered}
Y\left(k_{1}, k_{2}, \omega\right)=\jmath \omega \sum_{q=1}^{(2 N+1)^{2}}\left[\sum _ { r = 1 } ^ { 8 ( 2 N + 1 ) ^ { 2 } } A ^ { ( r ) } \left(D_{3}^{q,(r)}\right.\right. \\
\left.\left.-\epsilon_{0}|\kappa| \phi^{q,(r)}\right) e^{-j k_{3}^{(r)} h}\right] \\
\times p_{1} \operatorname{sinc}\left(\left(k_{1}+G_{1}^{q}\right) \frac{p_{1}}{2}\right) p_{2} \operatorname{sinc}\left(\left(k_{2}+G_{2}^{q}\right) \frac{p_{2}}{2}\right)
\end{gathered}
$$

where $\kappa=\sqrt{\left(k_{1}+G_{1}^{q}\right)^{2}+\left(k_{2}+G_{2}^{q}\right)^{2}}$.

\section{A. Frequency dependent, viscoelastic loss model}

The degree of mechanical loss is usually expressed in terms of a dimensionless loss tangent $\tan \delta$ [13], a mechanical factor $Q$, or an attenuation coefficient $\alpha$ [14]. Frequency dependent loss is introduced into the ceramic phase via $\tan \delta$ using $\tan \delta \propto w^{\gamma}$, with $\gamma \in[1,2]$. Loss is introduced into the polymer by defining complex Lamé coefficients and deriving expressions for their imaginary parts in terms of the natural frequency and the attenuation coefficients. The degree of shear loss is then varied by multiplying the shear attenuation coefficient by a parameter $\zeta \in[1, \infty)$. The effect that a high shear attenuation polymer phase has on the frequency band gap surrounding the thickness mode of the transducer can then be investigated by simply varying $\zeta$.

\section{B. Implementation}

The vibrational modes of the transducer must satisfy the system of equations $X A^{(r)}=Q$, where $X(k, \omega)$ is an $8(2 N+$ $1)^{2} \times 8(2 N+1)^{2}$ matrix and $Q(k)$ is a column vector of length $8(2 N+1)^{2}$. Unfortunately the matrix $X$ is illconditioned, mainly due to the exponential terms which arise when calculating the boundary conditions at the mechanical and piezoelectric discontinuities. To help obviate this problem, the parameters are scaled to balance the matrix entries [12]. There are also numerical instabilities when the determinant of $X$ approaches zero, due to this ill-conditioning and Tikhonov regularisation [15] has been used to circumvent such issues [12]. This method converts the matrix to a real, symmetric form and then translates the eigenvalues by a small amount, $\mu$, along the real axis, away from the origin. The determinant of the resultant matrix is very large and the modes are identified by the minima in the cost function surface $\log \left|X^{*} X+\mu I\right|$, parameterised by the angular frequency, $\omega \in \mathbb{R}$, and wavenumbers $k_{1}, k_{2} \in \mathbb{C}$. A particular mode for a 2-2 design is obtained by setting $k_{2}=0, k_{1}$ to a real number (initially) and then searching the cost function surface in the $\omega$ direction until each local minima is found. These interim minima are used as the initial values for a search in the direction of the imaginary part of $k_{1}$, although here the algorithm stops at the first local minimum. This orthogonal stepping procedure is repeated a set number of times and the whole process is then performed for a range of $k_{1}$ values [12].

\section{RESULTS}

The first section shows a typical lossy dispersion curve for a 2-2 composite transducer. This is followed by a comparison of the dispersion curves obtained by the PWE method, a FE method and experimentally measured data for a 1-3 composite transducer. The second section discusses the damping of unwanted modes by the use of a polymer phase containing high shear attenuation.

\section{A. Comparison to FE Model and Experimental Data}

Fig. 1 shows a lossy dispersion diagram where each modal point has been shaded according to the size of the imaginary part of the wavenumber $\left(k_{1}\right)$, with the lighter shades corresponding to the highly attenuated modes. It can be seen that the degree of attenuation increases with frequency and eventually leads to each mode being cut off. It will be seen later that the experimental data displays mode cut-off and hence the inclusion of loss into the PWE method has considerably improved its predictive capabilities. The PWE method was then compared to experimental and FE modelling data from a 1-3 composite transducer composed of PZT5H ceramic (35\%) 


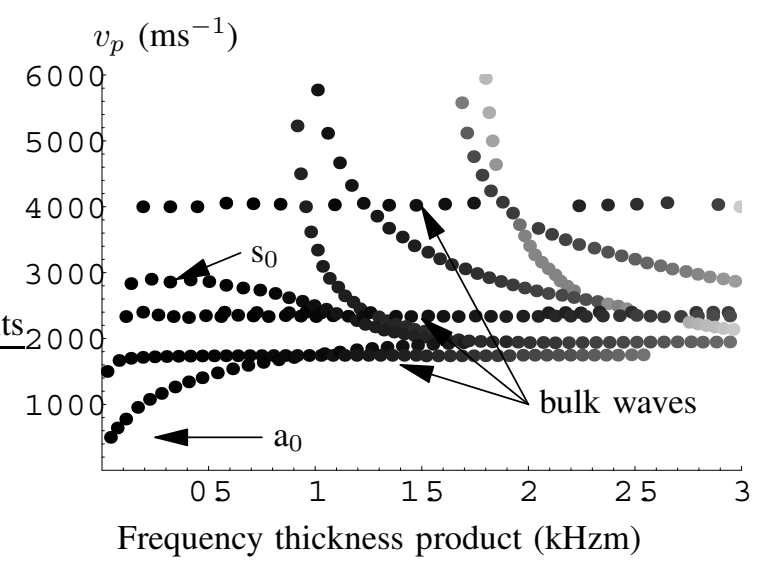

Fig. 1. Phase velocity $\left(v_{p}\right)$ versus frequency thickness product for a 22 composite transducer. The points are shaded according to the degree of attenuation given by the magnitude of the imaginary part of the wavenumber $k_{1}$.

\begin{tabular}{|c|c|c|}
\hline Data Values & Constant & Hardset \\
\hline Shear modulus (real part) & $G^{\prime}\left(\mathrm{kg} \mathrm{m}^{-1} \mathrm{~s}^{-2}\right)$ & $1.57 \times 10^{9}$ \\
Young's modulus (real part) & $E^{\prime}\left(\mathrm{kg} \mathrm{m}^{-1} \mathrm{~s}^{-2}\right)$ & $4.28 \times 10^{9}$ \\
Density & $\rho\left(\mathrm{kg} \mathrm{m}^{-3}\right)$ & $1.149 \times 10^{3}$ \\
Dielectric constant & $\epsilon$ & 4 \\
1-1 elastic constant & $c_{11}$ & $7.1977 \times 10^{9}$ \\
4-4 elastic constant & $c_{44}$ & $1.5739 \times 10^{9}$ \\
Shear attenuation coefficient & $\alpha_{0}^{s}(\mathrm{db} / \mathrm{m})$ & 356 \\
Longitudinal attenuation coefficient & $\alpha_{0}^{l}(\mathrm{db} / \mathrm{m})$ & 139 \\
Frequency of measurement & $f_{0}(\mathrm{MHz})$ & 0.5 \\
\hline
\end{tabular}

TABLE I

PHYSICAL PROPERTIES OF THE POLYMER HY1300/CY1301 HARDSET [16]

\begin{tabular}{|c|c|c|c|}
\hline- & Constant & Units & Value \\
\hline elastic constants & $c_{11}$ & $\mathrm{Nm}^{-2}$ & $12.72 \times 10^{10}$ \\
& $c_{12}$ & $\mathrm{Nm}^{-2}$ & $8.02 \times 10^{10}$ \\
& $c_{13}$ & $\mathrm{Nm}^{-2}$ & $8.47 \times 10^{10}$ \\
& $c_{33}$ & $\mathrm{Nm}^{-2}$ & $11.74 \times 10^{10}$ \\
& $\epsilon_{33}$ & - & 1700 \\
dielectric constant & $\epsilon_{11}$ & - & 1470 \\
Piezoelectric constant & $h$ & $\mathrm{~V} \mathrm{~m}^{-1}$ & $2.6 \times 10^{9}$ \\
density & $\rho_{b}$ & $\mathrm{~kg} \mathrm{~m}^{-3}$ & $7.5 \times 10^{3}$ \\
Piezoelectric stress & $e_{33}$ & $\mathrm{C} \mathrm{m}^{-2}$ & 23.3 \\
coefficients & $e_{31}$ & $\mathrm{C} \mathrm{m}$ & -6.5 \\
loss tangent & $\tan \delta$ & - & $1 / 65$ \\
\hline
\end{tabular}

TABLE II

PHYSICAL PROPERTIES OF THE CERAMIC PHASE PZT5H [16]

and HY1300/CY1301 hardset polymer (65\%) [2] (see Fig. 2, Table I and Table II). In addition to predicting the Lamb modes the PWE method also predicts the presence of bulk waves and interpillar modes. These latter waves are driven by the spacing between adjacent pillars and diagonally opposite pillars and are displayed as constant wavelength 'loadlines' in the dispersion diagram shown in Fig. 2. There is good agreement with the experimental data although the FE model predicts a larger frequency range for the $a_{0}$ mode than was observed experimentally. The PWE method is computationally less intensive than the FE model and its strength lies in providing a fast, qualitative prediction of the transducer's characteristics.

\section{B. Damping of unwanted lateral modes}

This section investigates a 2-2 composite transducer composed of PZT5H (70\%) ceramic and HY1300/CY1301 (30\%) hardset polymer. To simulate the effect of increasing the shear attenuation, the scaling parameter $\zeta$ is used to scale the shear attenuation coefficient. An alternative approach to

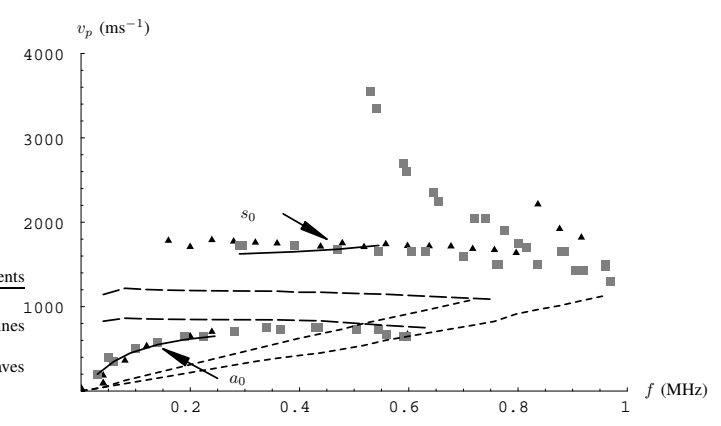

Fig. 2. Phase velocity $\left(v_{p}\right)$ versus frequency $(f)$ for a 1-3 composite transducer using the PWE method (triangles), FE modelling (squares) and experimental data (solid line). The long and short dashed lines represent the bulk modes and loadlines respectively.

calculating the dispersion curves is to utilise the electrical behaviour of the transducer. By plotting the conductance $(G)$ as a function of frequency, each of the maxima can be analysed in turn and the type of mode that is being supported at this frequency classified (see Fig. 3). The standard classification

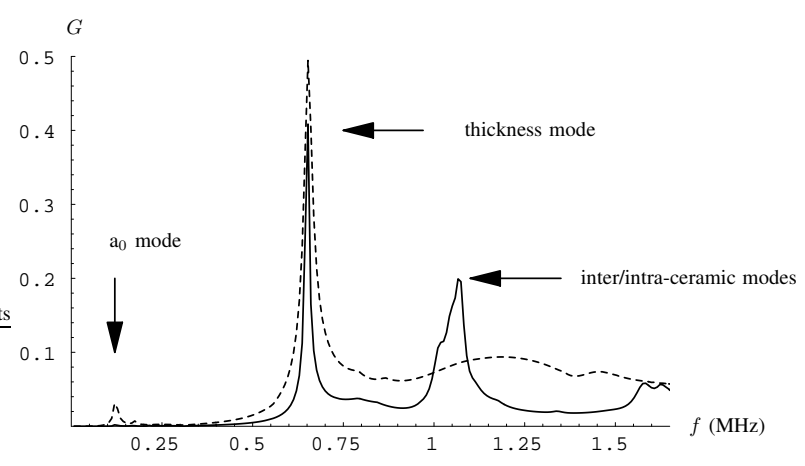

Fig. 3. Conductance $(G)$ versus frequency $(f)$ for a 2-2 composite transducer. The solid and dashed lines represent low shear attenuation $(\zeta=1)$ and high shear attenuation respectively $(\zeta=20)$.

of the modes is problematic here as the supporting medium is heterogeneous, anisotropic, lossy and piezoelectric. As such the descriptions of the waves in terms of their symmetry, or as Lamb, Rayleigh, bulk waves etc. are only pseudo-descriptions and the actual behaviour is far more complex. Identification of modes is aided by spatial and/or temporal plots of the displacement, the Poynting vector and the electrical potential. It transpires that the thickness mode can be identified as the central maximum in the admittance plot at around 0.65 MHz. The lower frequency maxima correspond to Lamb waves 
whilst the inter/intra-ceramic modes are the first set of peaks to the right of the thickness mode at around $1 \mathrm{MHz}$. The advantages of plotting the conductance is that the relative importance of each mode can be seen and in this way it eradicates any spurious points found in the dispersion diagram. The electrical conductance of the transducer can also be used to examine the effect of varying the loss in the passive phase (see Fig. 3). It can clearly be seen that the higher shear attenuation passive phase damps out the unwanted inter/intra-ceramic modes and attenuates the low frequency Lamb modes. To illustrate the
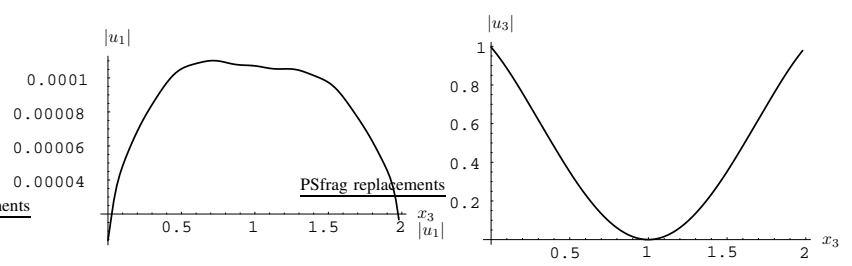

Fig. 4. Normalised displacement of a 2-2 composite transducer at the thickness mode.

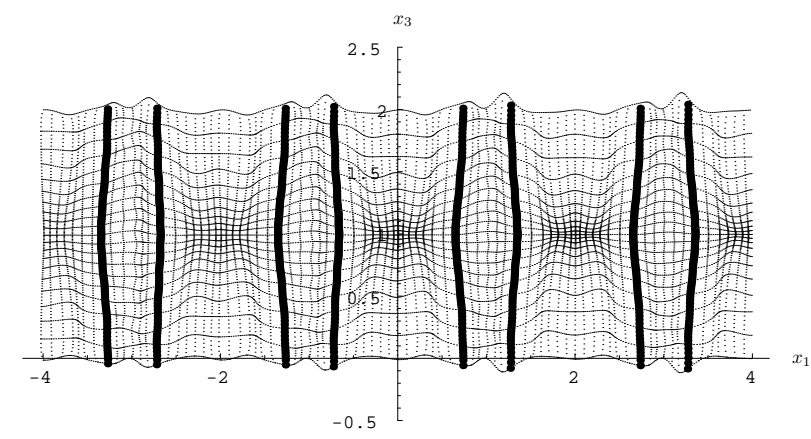

Fig. 5. Normalised Poynting vector $\left(P_{j}\right)$ in the $x_{3}-x_{1}$ plane of a $2-2$ composite transducer at the thickness mode.

mode identification process, the behaviour of the device at the thickness mode is examined. The displacement of the device is shown in Fig. 4 and it can be seen that $u_{1}$ is negligible compared to $u_{3}$, which has its largest values at the faces of the transducer. Examination of the temporal evolution of the device showed that the ceramic pillars are moving vertically with very little motion in the $x_{1}$ direction, and the polymer is being pulled sideways with very little motion in the $x_{3}$ direction. The in-plane Poynting vector can be viewed by proportionally displacing the $x_{1}$ and $x_{3}$ components to show where the energy is stored. For example, Fig. 5 shows that the energy is distributed throughout the transducer, primarily in the thickness direction. The symmetrical displacement profile in both directions, the large amplitude of oscillation and the dominant displacement being in the $x_{3}$ direction all point to this being the thickness mode.

\section{CONCLUSIONS}

The plane wave expansion (PWE) method is a frequency domain approach to studying the modal behaviour of periodic piezoelectric composite transducers. This paper has shown that the method can be extended to incorporate frequency dependent loss in both phases. The subsequent minimisation procedure culminates in a dispersion diagram which can highlight the attenuation of the modes as the frequency increases. Although the standard classification of the modes is difficult, as the supporting medium is heterogeneous, anisotropic, lossy and piezoelectric, pseudo-descriptions of the main supported modes of vibrations using spatial and/or temporal plots of the displacement and the Poynting vector can be given. Good agreement between the PWE method, FE method and experimental data was shown. Electrical device characteristics, for low and high shear attenuation in the passive phase, were compared and this showed that the use of a high shear loss polymer phase results in an improved stop band gap around the thickness mode.

\section{REFERENCES}

[1] G. Hayward and J. Hyslop, "Determination of Lamb Wave Dispersion in Data in Lossy Anisotropic Plates Using Time Domain Finite Element Analysis. Part 1: Theory and Experimental Verification", IEEE Trans. UFFC., vol. 53, no. 2, pp. 443-448, Feb. 2006.

[2] G. Hayward and J. Hyslop, "Determination of Lamb Wave Dispersion in Data in Lossy Anisotropic Plates Using Time Domain Finite Element Analysis. Part 2: Application to 2-2 and 1-3 Piezoelectric Composite Transducer Arrays", IEEE Trans. UFFC., vol. 53, no. 2, pp. 449-455, Feb. 2006.

[3] H.P. Savakus, K.A. Klicker and R.E. Newnham, "PZT-Epoxy Piezoelectric Transducer: A Simplified Fabrication Procedure", Mat. Res. Bull., vol. 16, pp. 677-680, 1981.

[4] T.R. Gururaja, W.A. Schulze, L.E. Cross, R.E. Newnham, B.A. Auld and Y.J. Wang, "Piezoelectric composite materials for ultrasonic transducer applications. Part 1: Resonant modes of vibration of PZT rod-polymer composites", IEEE Trans. Sonics and Ultrasonics, vol. SU-22, no. 4, pp. 481-498, July 1985.

[5] A.C.S. Parr, A. Troge, R.L. O'Leary, R.A. Pethrick and G. Hayward, "Performance of Periodic Piezoelectric Composite Arrays Incorporating a Passive Phase Exhibiting Anisotropic Properties", Proceedings of the 2005 IEEE International Ultrasonics Symposium, pp. 1073-1076, 2005.

[6] Y. Shui, X. Geng and Q.M. Zhang, "Theoretical Modelling of Resonant Modes of Composite Ultrasonic Transducers", IEEE Trans. UFFC., vol. 42, no. 4, pp. 766-773, July 1995.

[7] D. Certon, F. Patat, F. Levassort, G. Feuillard and B. Karlsson, "Lateral Resonances in 1-3 Piezoelectric Periodic Composites: Modelling and Experimental Results", JASA, vol. 101, no. 4, pp. 2043-2051, Apr. 1997.

[8] G. Hayward and J.A. Hossack, "Unidimensional Modelling of 1-3 Composite Transducers", JASA, vol. 88, no. 2, pp. 599-607, 1990.

[9] S. Ballandras, M. Wilm, P. Edoa and A. Soufyane, "Finite-Element Analysis of Periodic Piezoelectric Transducers", JASA, vol. 93, no. 1, pp. 702-711, 2003.

[10] M. Wilm, S. Ballandras, V. Laude, and T. Pastureaud, "A Full 3D PlaneWave-Expansion Model for 1-3 Piezoelectric Composite Structures", JASA, vol. 112, no. 3, pp. 943-952, Sept. 2002.

[11] “ANSI/IEEE Standard on Piezoelectricity", IEEE Trans. UFFC., vol. 43, no. 5, pp. 717-771, Sept. 1996.

[12] L-A. Orr and A.J. Mulholland, "Modelling of 1-3 Composite Transducers using the Plane Wave Expansion Method" Research Report no.18, Dept of Mathematics, University of Strathclyde, Glasgow, UK, 2005.

[13] N.G. McCrum, B.E. Read and G. Williams, Anelastic and Dielectric Effects in Polymeric Solids, John Wiley and Sons, 1967.

[14] R.S. Lakes, Viscoelastic Solids, CRC Press, London, 1999.

[15] A. Neumaier, "Solving Ill-conditioned and Singular Linear Systems; A Tutorial on Regularization", IEEE Trans. UFFC., vol. 44, no. 5, pp. 10181026, Sept. 1998.

[16] R.L. O'Leary, G. Smillie, G. Hayward and A.C.S. Parr, CUE Materials Database, Centre for Ultrasonic Engineering, University of Strathclyde, Glasgow, Scotland, June 2002. www.cue.ac.uk 